\title{
A Birch and Swinnerton-Dyer conjecture for the Mazur-Tate circle pairing
}

\author{
Massimo Bertolini \\ Henri Darmon
}

September 5, 2007

\begin{abstract}
Let $E$ be an elliptic curve over $\mathbb{Q}$ attached to a newform $f$ of weight two on $\Gamma_{0}(N)$, and let $K$ be a real quadratic field in which all the primes dividing $N$ are split. This note relates the canonical $\mathbb{R} / \mathbb{Z}$-valued "circle pairing" on $E(K)$ defined by Mazur and Tate [MT1] to a period integral $I^{\prime}(f, K)$ defined in terms of $f$ and $K$. The resulting conjecture can be viewed as an analogue of the classical Birch and Swinnerton-Dyer conjecture, in which $I^{\prime}(f, K)$ replaces the derivative of the complex $L$-series $L(f, K, s)$, and the circle pairing replaces the Néron-Tate height. It emerges naturally as an archimedean fragment of the theory of anticyclotomic $p$-adic $L$-functions developed in [BD1], and has been tested numerically in a variety of situations. The last section formulates a conjectural variant of a formula of Gross, Kohnen and Zagier [GKZ] for the Mazur-Tate circle pairing.
\end{abstract}

\section{Contents}

1 The circle pairing 2

2 Derived periods 5

3 The conjecture $\quad 12$

4 Numerical evidence $\quad 15$

5 A Gross-Kohnen-Zagier formula 18

\section{Introduction}

Let $E$ be an elliptic curve over $\mathbb{Q}$ of conductor $N$. Fix a real quadratic field $K \subset \mathbb{R}$, and let $\varepsilon_{K}>1$ denote the fundamental unit of $K$ of positive norm. Write $E(K)^{+}$(resp. $E(K)^{-}$) for the subgroup of the Mordell-Weil group $E(K)$ 
on which the generator of $\operatorname{Gal}(K / \mathbb{Q})$ acts as multiplication by 1 (resp. -1 ), so that $E(K)^{+}=E(\mathbb{Q})$.

In [MT1], §3.5, Mazur and Tate define (under the assumption that $K$ has class number one) a canonical $\mathbb{Z}$-bilinear "circle pairing"

$$
E(K)^{-} \times E(\mathbb{Q}) \longrightarrow \mathbb{R} /\left(\mathbb{Z} \cdot \log \varepsilon_{K}\right)
$$

and raise the question of what meaning can be ascribed to this pairing. (Cf. remark 3.5.3 of [MT1].)

By the results of $[\mathrm{W}],[\mathrm{TW}]$ and $[\mathrm{BCDT}]$, the elliptic curve $E$ is known to be modular; let $f$ be the associated normalised eigenform of weight 2 on $\Gamma_{0}(N)$. This note formulates a conjecture relating the circle pairing of (1) to a period integral $I^{\prime}(f, K)$ defined in terms of $f$ and $K$. Conjecture 3.1 of section 3 should be viewed as an analogue of the classical Birch and Swinnerton-Dyer conjecture, in which $I^{\prime}(f, K)$ replaces the derivative of the complex $L$-series $L(f, K, s)$, and the circle pairing replaces the Néron-Tate height. It emerges naturally as an archimedean fragment of the theory of anticyclotomic $p$-adic $L$-functions developed in [BD1], and has been tested numerically in a variety of situations which are described in section 4 . Section 5 formulates a conjectural variant of a formula of Gross, Kohnen and Zagier [GKZ] for the Mazur-Tate circle pairing whose proof would give strong evidence for conjecture 3.1.

\section{The circle pairing}

For any place $v$ of $K$, let $K_{v}$ denote the completion of $K$ at $v$ and let $\mathcal{O}_{v}$ (if $v$ is non-archimedean) be the ring of integers of $K_{v}$. Let

$$
U_{v}= \begin{cases}\mathcal{O}_{v}^{\times} & \text {if } v \text { is non-archimedean; } \\ \{1\} & \text { if } v \text { is real. }\end{cases}
$$

Denote by $\operatorname{Div}^{0}\left(E\left(K_{v}\right)\right) \times \operatorname{Div}^{0}\left(E\left(K_{v}\right)\right)$ the set of pairs of degree 0 divisors on $E\left(K_{v}\right)$ with disjoint supports. The classical Néron-Tate canonical height on $E(K)$ is defined in terms of the local Néron symbols

$$
[,]_{v}: \operatorname{Div}^{0}\left(E\left(K_{v}\right)\right) \times \operatorname{Div}^{0}\left(E\left(K_{v}\right)\right) \longrightarrow K_{v}^{\times} / U_{v}
$$

characterised uniquely (cf. Theorem 3 and remark (d) following its proof in $[\mathrm{Ne}])$ by the following properties:

1. The function $[,]_{v}$ is biadditive and symmetric;

2. For any principal divisor $(f)$, and any divisor $D$ with support disjoint from that of $(f)$,

$$
[(f), D]_{v}=f(D) \quad\left(\bmod U_{v}\right)
$$

3. The symbol $[,]_{v}$ is translation-invariant, i.e.,

$$
\left[D_{1}^{x}, D_{2}^{x}\right]_{v}=\left[D_{1}, D_{2}\right], \text { for all } x \in E\left(K_{v}\right),
$$

where $D_{i}^{x}$ denotes the translate of the divisor $D_{i}$ by the point $x$; 
4. For fixed $D$, the function $x \mapsto\left[(x)-\left(x_{0}\right), D\right]_{v}$ is bounded on bounded subsets of $E\left(K_{v}\right)-\operatorname{supp}(D)$.

The symbol (2) is defined using arithmetic intersection theory on the Néron model of $E$ over $\mathcal{O}_{v}$ when $v$ is non-archimedean, and using capacity theory (Green's functions) on $E\left(K_{v}\right)$ when $v$ is archimedean. (Cf. [Ne] and the discussion in $\S 2$ and 3 of [MT1].)

Let $\mathbb{A}_{K}^{\times} \subset \prod_{v} K_{v}^{\times}$denote the group of idèles of $K$. It is convenient to package the local symbols $[,]_{v}$ in the obvious way into an "idèlic symbol"

$$
[,]: \operatorname{Div}^{0}(E(K)) \times \operatorname{Div}^{0}(E(K)) \longrightarrow \mathbb{A}_{K}^{\times} /\left(\prod_{v} U_{v}\right) .
$$

Consider the quotient $C_{K}:=\mathbb{A}_{K}^{\times} /\left(\prod_{v} U_{v}\right) K^{\times}$of the idèle class group of $K$. If $(f)$ is a principal divisor on $E(K)$, and $D$ an arbitrary degree 0 divisor with support disjoint from that of $(f)$, note that

$$
[(f), D]=(f(D)) \text { belongs to } K^{\times},
$$

so that [, ] descends to a $C_{K}$-valued pairing

$$
\langle,\rangle: E(K) \times E(K) \longrightarrow C_{K} .
$$

If $|\cdot|: C_{K} \longrightarrow\left(\mathbb{R}^{+}\right)^{\times}$denotes the homomorphism induced by the idèlic norm (which is trivial on $K^{\times}$by the product formula) then the classical Néron-Tate canonical height is given by the formula

$$
\langle P, Q\rangle_{N T}:=\log (|\langle P, Q\rangle|) .
$$

(Cf. prop (2.3.1) of [MT1].)

Let $C l(K)$ and $C l^{+}(K)$ denote the class group and narrow class group of $K$ respectively, and denote by $h$ and $h^{+}$their orders, so that

$$
h^{+}=\left\{\begin{aligned}
h & \text { if } \mathcal{O}_{K} \text { has a unit of negative norm; } \\
2 h & \text { otherwise. }
\end{aligned}\right.
$$

Let $C_{K}^{-} \subset C_{K}$ denote the kernel of the idèlic norm on $C_{K}$. It is a compact group, sitting in the middle of an exact sequence

$$
0 \longrightarrow \mathbb{R} /\left(\mathbb{Z} \cdot \log \varepsilon_{K}\right) \stackrel{\alpha}{\longrightarrow} C_{K}^{-} \stackrel{\beta}{\longrightarrow} C l^{+}(K) \longrightarrow 0,
$$

where $\beta$ is the canonical map which assigns to the idèle class $c$ the corresponding narrow ideal class, and $\alpha$ sends $x$ to the class of the idèle $\left(1, \ldots, 1, e^{x}, e^{-x}\right)$.

Let $\tau$ be the generator of $\operatorname{Gal}(K / \mathbb{Q})$, and let $\eta: C_{K} \longrightarrow C_{K}^{-}$be the homomorphism sending $x$ to $x / x^{\tau}$. The information lost in the passage from $\langle$,$\rangle to$ $\langle,\rangle_{N T}$ is encoded for the most part in the Mazur-Tate circle pairing

$$
\langle,\rangle_{\text {circle }}: E(K) \times E(K) \longrightarrow C_{K}^{-}, \quad \text { defined by } \quad\langle P, Q\rangle_{\text {circle }}=\eta(\langle P, Q\rangle) \text {. }
$$


The terminology arises from the fact that $\langle,\rangle_{\text {circle }}$ takes its values in $C_{K}^{-}$, an extension of a finite group by a circle. The Mazur-Tate circle pairing is a $\mathbb{Z}$-bilinear form on $E(K)$ satisfying the following Galois equivariance property:

$$
\left\langle P^{\tau}, Q^{\tau}\right\rangle_{\text {circle }}=-\langle P, Q\rangle_{\text {circle }}
$$

In fact, the subspaces $E(\mathbb{Q})$ and $E(K)^{-}$are isotropic for $\langle,\rangle_{\text {circle }}$ for if $P$ and $Q$ belong to the same eigenspace for $\tau$, then the idèle class $\langle P, Q\rangle$ is fixed by $\tau$ and hence is in the kernel of $\eta$. For further discussion of the Mazur-Tate circle pairing, see [MT1], [MT2], [Ca], [Bo], and [Ha].

Let $r^{+}$and $r^{-}$denote the ranks of $E(\mathbb{Q})$ and $E(K)^{-}$respectively, and let $\left(P_{1}^{+}, \ldots, P_{r^{+}}^{+}\right)$and $\left(P_{1}^{-}, \ldots, P_{r^{-}}^{-}\right)$denote bases for $E(\mathbb{Q})$ and for $E(K)^{-}$respectively, modulo torsion. The pairing matrix attached to the Mazur-Tate circle pairing and to the basis $\left(P_{1}^{+}, \ldots, P_{r^{+}}^{+}, P_{1}^{-}, \ldots, P_{r^{-}}^{-}\right)$of $E(K) \otimes \mathbb{Q}$ is of the form

$$
\left(\begin{array}{cc}
0_{r^{+}} & M \\
M^{t} & 0_{r^{-}}
\end{array}\right)
$$

where $M$ is an $r^{+} \times r^{-}$matrix with entries in $C_{K}^{-}$given by

$$
M_{i j}=\left\langle P_{i}^{+}, P_{j}^{-}\right\rangle_{\text {circle }}
$$

It would be tempting to define the Mazur-Tate regulator in this context (or rather, a quantity akin to its square root) by the formula

$$
R_{\text {circle }}^{\frac{1}{2}} \stackrel{?}{=}\left\{\begin{array}{cc}
\operatorname{det}(M) & \text { if } r^{+}=r^{-} \\
0 & \text { otherwise. }
\end{array}\right.
$$

But since the Mazur-Tate pairing matrix has entries in a product of a finite group by a circle, which is not endowed with a ring structure, one is hard pressed to define its determinant in a sensible way - the one notable exception arising when $r^{+}=r^{-}=1$ !

Thus we set

$$
R_{\text {circle }}^{\frac{1}{2}}=\left\{\begin{array}{cc}
\left\langle P_{1}^{+}, P_{1}^{-}\right\rangle & \text {if } r^{+}=r^{-}=1 \\
0 & \text { otherwise. }
\end{array}\right.
$$

This definition (or lack thereof, in the higher-rank case) reflects a difficulty in our variant of the Birch and Swinnerton-Dyer conjecture which arises on two levels:

1. On the "arithmetic side", we are unable to propose an interesting definition of a regulator for a pairing matrix whose entries take values in a circle group, when $r^{+}$or $r^{-}$is $>1$.

2. On the "analytic side", as will be seen in the next section, the derived period $I^{\prime}(f, K)$ defined there and playing the role of $L^{\prime}(f, K, 1)$ is conjectured to vanish when $r^{+}$or $r^{-}$is strictly greater than one, and no obvious candidate for a "higher derived period" playing the role of higher derivatives of $L$-series has emerged from the formalism described in section 2 . 


\section{Derived periods}

Let

$$
\omega_{f}:=2 \pi i f(z) d z=\sum_{n>0} a_{n} q^{n} \frac{d q}{q}, \quad q=e^{2 \pi i z}
$$

denote the $\Gamma_{0}(N)$-invariant differential on $\mathcal{H}$ associated to $f$.

Assume in the rest of the article that the real quadratic field $K$ satisfies

$$
\text { the discriminant of } K \text { is prime to } N \text {. }
$$

Definition 2.1 An algebra embedding

$$
\Psi: K \longrightarrow M_{2}(\mathbb{Q})
$$

is said to be optimal (with respect to $N$ ) if

$$
\Psi(K) \cap M_{0}(N)=\Psi\left(\mathcal{O}_{K}\right),
$$

where $M_{0}(N)$ is the algebra of matrices with entries in $\mathbb{Z}$ which are uppertriangular modulo $N$.

Lemma 2.2 An optimal embedding of $K$ of level $N$ exists if and only if all the prime divisors of $N$ are split in $K / \mathbb{Q}$.

Proof: If $\Psi$ is an optimal embedding with respect to $N$, then the map

$$
\mathfrak{o}_{\Psi}: \mathcal{O}_{K} \longrightarrow \mathbb{Z} / N \mathbb{Z}
$$

which to $x$ associates the lower right-hand entry of $\Psi(x)$ (taken modulo $N$ ) is a ring homomorphism; such a homomorphism can only exist, in light of assumption (3), if all the primes dividing $N$ are split in $K / \mathbb{Q}$. Conversely, if this condition is satisfied then there is a cyclic ideal $\mathcal{N}$ of $\mathcal{O}_{K}$ of norm $N$. Choose a $\mathbb{Z}$-basis $\left(e_{1}, e_{2}\right)$ of $\mathcal{O}_{K}$ in such a way that $e_{1}$ belongs to $\mathcal{N}$. The action of $\mathcal{O}_{K}$ on itself by left multiplication, expressed in this basis, yields the desired optimal embedding. More precisely,

$$
\Psi(\alpha)=\left(\begin{array}{ll}
a & b \\
c & d
\end{array}\right), \quad \text { where }\left\{\begin{array}{l}
\alpha e_{1}=a e_{1}+c e_{2}, \\
\alpha e_{2}=b e_{1}+d e_{2} .
\end{array}\right.
$$

Motivated by lemma 2.2 assume that

$$
\text { all primes dividing } N \text { are split in } K / \mathbb{Q} \text {. }
$$

In that case one has

$$
\operatorname{sign}(E, K)=1
$$

where $\operatorname{sign}(E, K)$ denotes the sign in the functional equation for $L(E, K, s)$. In particular, the Birch and Swinnerton-Dyer conjecture predicts that $E(K)$ has even rank when assumption (5) holds.

Fix a ring homomorphism $\mathfrak{o}_{0}: \mathcal{O}_{K} \longrightarrow \mathbb{Z} / N \mathbb{Z}$ (or, what amounts to the same thing, a cyclic ideal ker $\mathfrak{o}_{0}$ of $\mathcal{O}_{K}$ of norm $N$, or a choice of square root of $\operatorname{Disc}(K)$ modulo $N)$. 
Definition 2.3 The optimal embedding $\Psi$ is said to be oriented (with respect to the choice of $\mathfrak{o}_{0}$ ) if the homomorphism $\mathfrak{o}_{\Psi}$ associated to it as in the proof of lemma 2.2 is equal to $\mathfrak{o}_{0}$.

Note that if $\Psi$ is an oriented optimal embedding, then so is the conjugate embedding $\alpha \Psi \alpha^{-1}$ for any $\alpha \in M_{0}(N)^{\times}$.

One may attach to an ideal class $\mathcal{C}$ of $K$ an oriented optimal embedding $\Psi_{\mathcal{C}}$ by choosing a representative ideal $\mathfrak{c} \in \mathcal{C}$ of norm prime to $N$, a $\mathbb{Z}$-basis $\left(e_{1}, e_{2}\right)$ for $\mathfrak{c}$ such that $e_{1}$ belongs to $\mathfrak{c} \cap \mathcal{N}$, and defining $\Psi$ as in (4). The resulting embedding is independent of the choice of $\mathfrak{c}$ and $\left(e_{1}, e_{2}\right)$ up to conjugation by $M_{0}(N)^{\times}$, so that the assignment $\mathcal{C} \mapsto \Psi_{\mathcal{C}}$ sets up a bijection

$$
\left\{\begin{array}{l}
\text { Ideal classes } \\
\text { of } K
\end{array}\right\} \stackrel{\simeq}{\longrightarrow}\left\{\begin{array}{l}
\text { Oriented optimal embeddings } \\
\text { of } \mathcal{O}_{K} \text { into } M_{0}(N)
\end{array}\right\} / M_{0}(N)^{\times} .
$$

If $\mathcal{C}$ is a narrow ideal class, one may also insist that the basis of $\mathfrak{c}$ be oriented, i.e., that

$$
\operatorname{det}\left(\begin{array}{ll}
e_{1} & \tau e_{1} \\
e_{2} & \tau e_{2}
\end{array}\right)>0
$$

Then $\Psi_{\mathcal{C}}$ becomes well defined up to conjugation by $\Gamma_{0}(N)$, the group of elements of $M_{0}(N)^{\times}$of positive determinant, and the assignment $\mathcal{C} \mapsto \Psi_{\mathcal{C}}$ sets up a bijection

$$
\left\{\begin{array}{l}
\text { Narrow ideal classes } \\
\text { of } K
\end{array}\right\} \stackrel{\simeq}{\longrightarrow}\left\{\begin{array}{l}
\text { Oriented optimal } \\
\text { embeddings } \\
\text { of } \mathcal{O}_{K} \text { into } M_{0}(N)
\end{array}\right\} / \Gamma_{0}(N) .
$$

Thanks to these identifications, the set of oriented optimal embeddings of $\mathcal{O}_{K}$ into $M_{0}(N)$, taken up to conjugation by $M_{0}(N)^{\times}\left(\right.$resp. $\left.\Gamma_{0}(N)\right)$ becomes a principal homogeneous space for the action of $\mathrm{Cl}(K)$ (resp. $\mathrm{Cl}^{+}(K)$ ). In particular, we have:

Lemma 2.4 There are exactly $h\left(\right.$ resp. $\left.h^{+}\right)$distinct oriented optimal embedding of $K$ of level $N$, up to conjugation by $M_{0}(N)^{\times}\left(\right.$resp. $\left.\Gamma_{0}(N)\right)$.

We now associate to $f$ and $K$ a canonical derived period $I^{\prime}(f, K) \in C_{K}^{-}$whose value is conjecturally related to the circle regulator of the previous section. The description is simpler when $K$ has narrow class number one, so we begin by treating this case, postponing the general case to the end of the section.

Let $\Psi$ be any optimal embedding of $K$ into $M_{2}(\mathbb{Q})$, which is unique up to conjugation in $\Gamma_{0}(N)$, by lemma 2.4. Let

$$
\gamma_{\Psi}:=\Psi\left(\varepsilon_{K}\right) \in \Gamma_{0}(N) .
$$

Choose an arbitrary base point $x$ in the extended Poincaré upper half plane $\mathcal{H}^{*}=\mathcal{H} \cup \mathbb{P}_{1}(\mathbb{Q})$. To this data is associated the period integral

$$
I_{\Psi, x}=\int_{x}^{\gamma_{\Psi} x} \omega_{f}
$$


It follows directly from the $\Gamma_{0}(N)$-invariance of $\omega_{f}$ that the period $I_{\Psi, x}$ is independent of the choice of $x$. Hence one may, when it is convenient, suppress the base point $x$ from the notation and set $I_{\Psi}:=I_{\Psi, x}$. A direct computation also shows that, if $\alpha$ belongs to $\Gamma_{0}(N)$, then

$$
I_{\alpha \Psi \alpha^{-1}}=I_{\alpha \Psi \alpha^{-1}, x}=I_{\Psi, \alpha^{-1} x}=I_{\Psi}
$$

Hence $I_{\Psi}$ depends only on the $\Gamma_{0}(N)$-conjugacy class of $\Psi$. Set

$$
I(f, K)=I_{\Psi}
$$

for any choice of optimal embedding $\Psi$ and base point $x$. By the assumption that $K$ has narrow class number one, this period does not depend on the choice of $\Psi$, thanks to lemma 2.4. Furthermore, this canonical period is related to special values of $L$-series as follows:

Lemma 2.5 The period $I(f, K)$ vanishes if and only if $L(f, K, 1)=0$.

Sketch of proof. A formula which is spelled out precisely in [Po] (see also chapter II of [GKZ]) implies that

$$
I(f, K)^{2} \doteq L(f, K, 1)
$$

where the symbol $\doteq$ denotes equality up to an explicit non-zero fudge factor. The result follows from this.

Assume henceforth that $L(f, K, 1)=0$, so that

$$
I(f, K)=0 .
$$

The group $\Psi\left(K^{\times}\right)$acting by Möbius transformations on $\mathbb{P}_{1}(\mathbb{C})$ has two fixed points $x_{\Psi}$ and $y_{\Psi}$ in $\mathbb{P}_{1}(\mathbb{R})$. Order these fixed points in such a way that $\gamma_{\Psi}$ has $x_{\Psi}$ as a repulsive fixed point, and $y_{\Psi}$ as an attractive fixed point.

Under assumption (9) it becomes natural to consider the following "derived period integral"

$$
I_{\Psi, x}^{\prime}=\int_{x}^{\gamma_{\Psi} x} \log \left(\frac{z-x_{\Psi}}{z-y_{\Psi}}\right) \omega_{f}
$$

To analyse the dependence of this quantity on the choice of base point $x \in \mathcal{H}^{*}$, note that

$$
I_{\Psi, x}^{\prime}-I_{\Psi, y}^{\prime}=\int_{x}^{y} \log \left(\frac{z-x_{\Psi}}{z-y_{\Psi}}\right) \omega_{f}-\int_{\gamma_{\Psi} x}^{\gamma_{\Psi} y} \log \left(\frac{z-x_{\Psi}}{z-y_{\Psi}}\right) \omega_{f} .
$$

Performing the change of variable $w=\gamma_{\Psi}^{-1} z$ in the second term, and noting that

$$
\left(\frac{\gamma_{\Psi} w-x_{\Psi}}{\gamma_{\Psi} w-y_{\Psi}}\right)=\varepsilon_{K}^{2} \cdot\left(\frac{w-x_{\Psi}}{w-y_{\Psi}}\right)
$$

yields

$$
I_{\Psi, x}^{\prime}-I_{\Psi, y}^{\prime}=2 \log \varepsilon_{K} \cdot \int_{x}^{y} \omega_{f}
$$


Thus the value of $I_{\Psi, x}^{\prime}$ depends strongly on the choice of $x$. One resolves this ambiguity by requiring that

$$
\text { the base point } x \text { belongs to } \mathbb{P}_{1}(\mathbb{Q}) \subset \mathcal{H}^{*} \text {. }
$$

A theorem of Manin and Drinfeld [Man] asserts that the subgroup of $\mathbb{C}$ generated by the expressions of the form $\int_{x}^{y} \omega_{f}$, where $x$ and $y$ belong to $\mathbb{P}_{1}(\mathbb{Q})$, is a lattice $\Lambda_{f}$ which is commensurable to the Néron lattice of $E$. This result is crucial in our definition of the derived period, for it implies

Lemma 2.6 The natural image of $I_{\Psi, x}^{\prime}$ in $\mathbb{C} / 2 \Lambda_{f} \log \varepsilon_{K}$ is independent of the choice of $x \in \mathbb{P}_{1}(\mathbb{Q})$. Furthermore it depends only on the $\Gamma_{0}(N)$-conjugacy class of $\Psi$.

Proof. The first assertion follows directly from (11). By a slight abuse of notation, we may therefore denote by $I_{\Psi}^{\prime}$ the natural image of $I_{\Psi, x}^{\prime}$ in $\mathbb{C} / 2 \Lambda_{f} \log \varepsilon_{K}$, for any choice of base point $x \in \mathbb{P}_{1}(\mathbb{Q})$. To prove the second assertion, let $\alpha=\left(\begin{array}{ll}a & b \\ c & d\end{array}\right)$ be an element of $\Gamma_{0}(N)$. Then a direct calculation shows that

$$
I_{\alpha \Psi \alpha^{-1}, \alpha x}^{\prime}-I_{\Psi, x}^{\prime}=c_{\alpha} \cdot I(f, K),
$$

where

$$
c_{\alpha}=\log \frac{\left(\alpha z-\alpha x_{\Psi}\right)\left(z-y_{\Psi}\right)}{\left(\alpha z-\alpha y_{\Psi}\right)\left(z-x_{\Psi}\right)}=\log \left(\frac{c y_{\Psi}+d}{c x_{\Psi}+d}\right)
$$

is a constant independent of $z$. It follows from (9) that

$$
I_{\alpha \Psi \alpha^{-1}}^{\prime}=I_{\Psi}^{\prime} \quad\left(\bmod 2 \Lambda_{f} \cdot \log \varepsilon_{K}\right) .
$$

It is useful to supplement (15) by the following equation which describes the variation of $I_{\Psi}^{\prime}$ under conjugation of $\Psi$ by an element $\alpha \in M_{0}(N)^{\times}-\Gamma_{0}(N)$ of determinant -1 .

$$
I_{\alpha \Psi \alpha^{-1}}^{\prime}=\bar{I}_{\Psi}^{\prime} \quad\left(\bmod 2 \Lambda_{f} \cdot \log \varepsilon_{K}\right) .
$$

Lemmas 2.4 and 2.6 make it possible (under the narrow class number one hypothesis) to associate to $f$ and $K$ a canonical "derived period"

$$
I^{\prime}(f, K) \in \mathbb{C} /\left(\Lambda_{f} \cdot \log \varepsilon_{K}\right),
$$

defined as the natural image of $I_{\Psi, x}^{\prime}$, for any choice of optimal embedding $\Psi$ of $\mathcal{O}_{K}$ in $M_{0}(N)$ and of base point $x \in \mathbb{P}_{1}(\mathbb{Q})$.

Let $\Omega^{+}$be the real period attached to $f$, defined as the unique positive generator of the lattice $\Lambda_{f} \cap \mathbb{R}$.

Lemma 2.7 The period $I^{\prime}(f, K)$ is fixed under complex conjugation. Its image in $\mathbb{C} /\left(\Lambda_{f} \cdot \log \varepsilon_{K}\right)$ belongs to the subgroup $\mathbb{R} /\left(\Omega^{+} \cdot \log \varepsilon_{K}\right)$. 
Proof. Let $\varepsilon \in \mathcal{O}_{K}^{\times}$be a unit of negative norm (which can be chosen for instance so that $\left.\varepsilon^{2}=\varepsilon_{K}\right)$ and let $\alpha=\Psi(\varepsilon) \in M_{0}(N)^{\times}$. Since $\alpha$ commutes with $\Psi\left(K^{\times}\right)$, we have

$$
\alpha \Psi \alpha^{-1}=\Psi, \quad \text { hence } I_{\alpha \Psi \alpha^{-1}}^{\prime}=I_{\Psi}^{\prime} \quad\left(\bmod 2 \Lambda_{f} \cdot \log \varepsilon_{K}\right) .
$$

The result now follows from (16).

Let $\operatorname{sign}(E, \mathbb{Q})= \pm 1$ denote the sign in the functional equation of the $L$ series $L(E / \mathbb{Q}, s)$. This sign is known to be the negative of the eigenvalue of the Atkin-Lehner involution acting on $\omega_{f}$.

Lemma 2.8 If $\operatorname{sign}(E, \mathbb{Q})=1$, then $I^{\prime}(f, K)=0$.

Proof. Let $\alpha_{N}=\left(\begin{array}{cc}0 & -1 \\ N & 0\end{array}\right)$ be the matrix in terms of which the Atkin-Lehner involution is defined. Since $\alpha_{N}$ normalises $\Gamma_{0}(N)$, the embedding $\alpha_{N} \Psi \alpha_{N}^{-1}$ is also an optimal embedding of $K$ of level $N$. A direct computation using the change of variables formula and the fact that $w_{N} f=-f$ shows that

$$
I_{\alpha_{N} \Psi \alpha_{N}^{-1}}^{\prime}=-I_{\Psi}^{\prime} \quad\left(\bmod 2 \Lambda_{f} \cdot \log \varepsilon_{K}\right) .
$$

Observe that $\alpha_{N} \Psi \alpha_{N}^{-1}$, although it is an optimal embedding, is not oriented; more precisely its orientation corresponds to the choice of the ideal $\overline{\mathcal{N}}=\tau \mathcal{N}$ rather than $\mathcal{N}$. Hence $\alpha_{N} \Psi \alpha_{N}^{-1}$ is $\Gamma_{0}(N)$-conjugate to the embedding $\Psi^{\prime}=\Psi \circ \tau$. Note that $\gamma_{\Psi^{\prime}}=\gamma_{\Psi}^{-1}$, so that $\left(x_{\Psi^{\prime}}, y_{\Psi^{\prime}}\right)=\left(y_{\Psi}, x_{\Psi}\right)$. Hence

$$
\begin{aligned}
I_{\alpha_{N} \Psi \alpha_{N}^{-1}}^{\prime} & =I_{\Psi^{\prime}}^{\prime}=\int_{x}^{\gamma_{\Psi}^{-1} x} \log \left(\frac{z-y_{\Psi}}{z-x_{\Psi}}\right) \omega_{f} \\
& =\int_{x}^{\gamma_{\Psi} x} \log \left(\frac{z-x_{\Psi}}{z-y_{\Psi}}\right) \omega_{f}=I_{\Psi}^{\prime} \quad\left(\bmod 2 \Lambda_{f} \cdot \log \varepsilon_{K}\right) .
\end{aligned}
$$

The result follows after comparing (17) and (18).

Because of lemma 2.8 , it is natural to assume that $\operatorname{sign}(E, \mathbb{Q})=-1$. The Birch and Swinnerton-Dyer conjecture then predicts - in light of assumption $(6)$ - that both $E(\mathbb{Q})$ and $E(K)^{-}$have odd rank.

We now turn to the derived period in the general case where $h^{+}$is not necessarily equal to 1 . In this case we define an invariant $J^{\prime}(f, K) \in C_{K}^{-}$, which, when $h^{+}=1$, is simply the class represented by the period $I^{\prime}(f, K)$ with the real period $\Omega^{+}$factored out. The definition of $J^{\prime}(f, K)$ (like the definition of the group to which it belongs) is best given adelically ${ }^{1}$.

It is worthwhile to allow more generality by introducing a quadratic character $\chi$ of the narrow ideal class group of $K$. The character $\chi$ is said to be even if it is trivial on the group of principal ideals (so that it factors through the class

\footnotetext{
${ }^{1}$ The reader may wish in a first reading to skip the rest of this section and jump directly to section 3 where the Birch and Swinnerton-Dyer-type conjecture relating $I^{\prime}(f, K)$ to the circle pairing is described.
} 
group of $K$ ) and is said to be odd otherwise (in which case it cuts out, by class field theory, a totally imaginary quadratic extension of $K$ ). We set the local sign $w$ to be 1 if $\chi$ is even, and -1 if $\chi$ is odd.

Let $\mathcal{C}_{1}, \ldots, \mathcal{C}_{h^{+}}$be a full set of representatives for the narrow ideal classes of $K$, and let $\Psi_{1}, \ldots, \Psi_{h^{+}}$denote representatives for the corresponding $\Gamma_{0}(N)$ conjugacy classes of oriented optimal embeddings of $\mathcal{O}_{K}$ into $M_{0}(N)$. Setting

$$
I(f, \chi)=\sum_{j=1}^{h^{+}} \chi\left(\mathcal{C}_{j}\right) I_{\Psi_{j}}
$$

one has (see the references given for lemma 2.5)

Lemma 2.9 The period $I(f, \chi)$ vanishes if and only if $L(E / K, \chi, 1)=0$.

The invariant $J^{\prime}(f, \chi)$ to be defined below (which we shall seek to interpret when $I(f, \chi)=0)$ plays the role of $L^{\prime}(E / K, \chi, 1)$ in the conjectures of section 3 . It is defined as a weighted linear combination of periods

$$
J_{\Psi_{j}}^{\prime} \in C_{K}^{-},
$$

indexed by the oriented optimal embeddings $\Psi_{j}$ of $\mathcal{O}_{K}$ into $M_{0}(N)$. Fix such an embedding $\Psi=\Psi_{j}$, and let $x_{\Psi}$ and $y_{\Psi} \in \mathbb{P}_{1}(K)$ be the fixed points of $\Psi\left(K^{\times}\right)$, normalised as before. Choose a rational function $g_{\Psi} \in K(x)$ satisfying

$$
\operatorname{Div}\left(g_{\Psi}\right)=\left(y_{\Psi}\right)-\left(x_{\Psi}\right)
$$

Note that this condition makes $g_{\Psi}$ well-defined up to multiplication by an element of $K^{\times}$.

Let $v$ be a prime of $K$, let $p$ be the rational prime which lies below it, and let $K_{v}$ and $k_{v}$ denote the corresponding completion and residue field. Reduction modulo $v$ gives a natural map $\mathbb{P}_{1}\left(K_{v}\right) \longrightarrow \mathbb{P}_{1}\left(k_{v}\right)$, denoted $x \mapsto \bar{x}$. Define a compact open subset $U_{\Psi, v} \subset \mathbb{P}_{1}\left(\mathbb{Q}_{p}\right)$ by the rule

$$
U_{\Psi, v}=\left\{\begin{array}{l}
\mathbb{P}_{1}\left(\mathbb{Q}_{p}\right) \text { if } p \text { is inert or ramified in } K / \mathbb{Q} . \\
\left\{t \in \mathbb{P}_{1}\left(\mathbb{Q}_{p}\right) \text { such that } \bar{t} \neq \bar{x}_{\Psi} \text { and } \bar{t} \neq \bar{y}_{\Psi}\right\}, \quad \text { otherwise. }
\end{array}\right.
$$

The key properties of $U_{\Psi, v}$ are summarised in the following two lemmas:

Lemma 2.10 If $t_{1}$ and $t_{2}$ belong to $U_{\Psi, v}$, then the cross ratio

$$
\left(\frac{t_{1}-y_{\Psi}}{t_{1}-x_{\Psi}}\right)\left(\frac{t_{2}-y_{\Psi}}{t_{2}-x_{\Psi}}\right)^{-1}=\frac{g_{\Psi}\left(t_{1}\right)}{g_{\Psi}\left(t_{2}\right)} \text { belongs to } \mathcal{O}_{v}^{\times} \text {. }
$$

Proof: If $v$ is inert or ramified in $K$, then the expression on the left belongs to $K_{v}^{\times}$and is of norm one. In any case, the reduction of the expression on the left modulo a uniformiser $\pi_{v}$ (i.e., its image in $\mathbb{P}_{1}\left(k_{v}\right)$ under the reduction map) is simply the cross-ratio of $\bar{t}_{1}, \bar{t}_{2}, \bar{x}_{\Psi}$, and $\bar{y}_{\Psi}$. By definition of $U_{\Psi, v}$, the points $\bar{t}_{1}$ and $\bar{t}_{2}$ are different from $\bar{x}_{\Psi}$ and $\bar{y}_{\Psi}$, and hence the lemma follows from the familiar properties of the cross ratio. 
Lemma 2.11 For all $\alpha \in \Gamma_{0}(N)$,

$$
U_{\alpha \Psi \alpha^{-1}, v}=\alpha U_{\Psi, v} .
$$

Proof: This follows directly from the fact that $\Gamma_{0}(N) \subset \mathbf{S L}_{2}\left(\mathcal{O}_{v}\right)$ acts naturally on $\mathbb{P}_{1}\left(K_{v}\right)$ and $\mathbb{P}_{1}\left(k_{v}\right)$ in a manner which is compatible with the reduction map

$$
\mathbb{P}_{1}\left(K_{v}\right) \longrightarrow \mathbb{P}_{1}\left(k_{v}\right) \text {. }
$$

For each finite place $v$ of $K$, fix a choice of "local base points" $t_{v} \in U_{\Psi, v}$, and choose a base point $t_{\infty} \in \mathbb{P}_{1}(\mathbb{Q})$. Write

$$
\underline{t}=\left(\ldots, t_{v}, \ldots ; t_{\infty}\right) \in \prod_{v} \mathbb{P}_{1}\left(\mathbb{Q}_{v}\right) \times \mathbb{P}_{1}(\mathbb{Q})
$$

for the infinite tuple corresponding to these choices.

Let $\Omega_{w}$ be the unique positive generator of $\Lambda_{f} \cap \mathbb{R}$ if $w=1$, and of $i^{-1} \Lambda_{f} \cap \mathbb{R}$ if $w=-1$. We define an element

$$
J_{\Psi, \underline{t}}^{\prime} \in C_{K}=\mathbb{A}_{K}^{\times} /\left(\prod_{v} U_{v}\right) K^{\times}
$$

by specifying each of its local components,

$$
\left(J_{\Psi, \underline{t}}^{\prime}\right)_{v} \in K_{v}^{\times} / \mathcal{O}_{v}^{\times}=\mathbb{Z}, \quad\left(J_{\Psi, \underline{t}}^{\prime}\right)_{\infty_{i}} \in \mathbb{R}^{\times}, \quad i=1,2 .
$$

Each $v$ of $K$ (either finite, or one of the two archimedean places $\infty_{1}$ or $\infty_{2}$ ) yields an embedding $K(z) \longrightarrow K_{v}(z)$ of rational function fields, and in this way $g_{\Psi}(z)$ gives rise to elements denoted $g_{\Psi}^{v}(z)$ in each $K_{v}(z)$. The components of $J_{\Psi, t}^{\prime}$ are defined as follows:

$$
\begin{aligned}
\left(J_{\Psi, \underline{t}}^{\prime}\right)_{v} & = \begin{cases}\operatorname{ord}_{v} g_{\Psi}^{v}\left(t_{v}\right) \cdot \operatorname{Re}\left(I_{\Psi}\right) \Omega_{+}^{-1} & \text { if } w=1 ; \\
\operatorname{ord}_{v} g_{\Psi}^{v}\left(t_{v}\right) \cdot \operatorname{Im}\left(I_{\Psi}\right) \Omega_{-}^{-1} & \text { if } w=-1 .\end{cases} \\
\left(J_{\Psi, \underline{t}}^{\prime}\right)_{\infty_{j}} & = \begin{cases}\exp \left(\operatorname{Re}\left(\int_{t_{\infty}}^{\gamma_{\Psi} t_{\infty}} \log g_{\Psi}^{\infty_{j}}(z) \omega_{f}\right) \Omega_{+}^{-1}\right) & \text { if } w=1 ; \\
\exp \left(\operatorname{Im}\left(\int_{t_{\infty}}^{\gamma_{\Psi} t_{\infty}} \log g_{\Psi}^{\infty_{j}}(z) \omega_{f}\right) \Omega_{-}^{-1}\right) & \text { if } w=-1 .\end{cases}
\end{aligned}
$$

We note that

1. The idèle class in $C_{K}$ corresponding to $J_{\Psi, \underline{t}}^{\prime}$ does not depend on the choice of $g_{\Psi}$. This is because multiplying $g_{\Psi}$ by a scalar in $K^{\times}$changes $J_{\Psi, \underline{t}}^{\prime}$ by the corresponding principal idèle.

2. For each non-archimedean place $v$, the local component $\left(J_{\Psi, t}^{\prime}\right)_{v}$ does not depend on the choice of base point $t_{v}$ that was made to define it. This follows directly from lemma 2.10 .

3. The archimedean components $\left(J_{\Psi, t}^{\prime}\right)_{\infty_{j}}$ do not depend on the choice of base point $t_{\infty} \in \mathbb{P}_{1}(\mathbb{Q})$ that was made to define them. This follows from the same manipulations as were used to derive formula (11). 
Finally we have

Lemma 2.12 The derived period $J_{\Psi, t}^{\prime}$ depends only on the $\Gamma_{0}(N)$-conjugacy class of $\Psi$.

Proof. Let $\Psi^{\prime}=\alpha \Psi \alpha^{-1}$ be an oriented optimal embedding of $\mathcal{O}_{K}$ into $M_{0}(N)$ which is conjugate to $\Psi$ under a matrix $\alpha \in \Gamma_{0}(N)$. The fixed points for this embedding are $\left(x_{\Psi^{\prime}}, y_{\Psi^{\prime}}\right)=\left(\alpha x_{\Psi}, \alpha y_{\Psi}\right)$, and we may set

$$
\begin{aligned}
g_{\Psi^{\prime}}(z) & =g_{\Psi}\left(\alpha^{-1} z\right) ; \\
\gamma_{\Psi^{\prime}} & =\alpha \gamma_{\Psi} \alpha^{-1} ; \\
U_{\Psi^{\prime}, v} & =\alpha U_{\Psi, v}, \quad \text { hence } t_{v}^{\prime}=\alpha t_{v} ; \\
t_{\infty}^{\prime} & =\alpha t_{\infty} ; \\
\underline{t}^{\prime} & =\left(\ldots, t_{v}^{\prime}, \ldots ; t_{\infty}^{\prime}\right) .
\end{aligned}
$$

With these choices it follows by a direct computation (using change of variables for the archimedean component) that

$$
\left(J_{\Psi, \underline{t}}^{\prime}\right)_{v}=\left(J_{\Psi^{\prime}, \underline{t}^{\prime}}^{\prime}\right)_{v} \quad \text { for all } v .
$$

The lemma now follows from the fact that $J_{\Psi, \underline{t}}^{\prime}$ does not depend on $\underline{t}$.

By an abuse of notation, let $J_{\Psi}^{\prime}$ denote the natural image of $J_{\Psi, \underline{t}}^{\prime}$ in $C_{K}^{-}$. As before, let $\mathcal{C}_{1}, \ldots, \mathcal{C}_{h^{+}}$be a full set of representatives for the narrow ideal classes of $K$, and let $\Psi_{1}, \ldots, \Psi_{h^{+}}$denote representatives for the corresponding $\Gamma_{0}(N)$-conjugacy classes of oriented optimal embeddings of $\mathcal{O}_{K}$ into $M_{0}(N)$. Since $J_{\Psi_{j}}^{\prime}$ depends only on $\mathcal{C}_{j}$ and not on the particular choice of representative, we may define

$$
\begin{aligned}
J^{\prime}(f, K) & =\sum_{j=1}^{h^{+}} J_{\Psi_{j}}^{\prime}, \quad(\text { with } w=1) . \\
J^{\prime}(f, \chi) & =\sum_{j=1}^{h^{+}} \chi\left(\mathcal{C}_{j}\right) J_{\Psi_{j}}^{\prime} .
\end{aligned}
$$

Question. In the simpler approach that was described when $h^{+}=1$, the fact that $I^{\prime}(f, K)$ was well-defined depended on the vanishing of $I(f, K)$ (cf. formula (13)). The adelic approach we adopted in the general case, involving a more careful integral normalisation of the factor $g_{\Psi}(t)$ appearing in the integrand, makes the derived periods $J_{\Psi}^{\prime}$ and $J^{\prime}(f, K)$ well-defined in $C_{K}^{-}$without any assumption on the vanishing of the corresponding periods $I_{\Psi}$ and $I(f, K)$. What meaning (if any) can be ascribed to $J^{\prime}(f, K)$ when $I(f, K) \neq 0$ ?

\section{The conjecture}

We begin by formulating the main conjecture in the special case where $h^{+}=1$, following the notations of the previous section that were introduced for this setting. Let $e$ denote the exponent of the torsion subgroup of $E(K)$. 
Conjecture 3.1 The derived period $I^{\prime}(f, K)$ is non-zero (and, even, of infinite order) if and only if $E(\mathbb{Q})$ and $E(K)^{-}$both have rank one. In that case

$$
I^{\prime}(f, K) \cdot t= \pm\left\langle P^{+}, P^{-}\right\rangle_{\text {circle }} \cdot \sqrt{\# \amalg} \cdot \Omega_{E}^{+} \cdot \prod_{p \mid N} c_{p} \quad\left(\bmod \Omega^{+} \cdot \frac{1}{e} \log \varepsilon_{K}\right),
$$

where

1. The points $P^{+}$and $P^{-}$are generators for $E(\mathbb{Q})$ and $E(K)^{-}$respectively, modulo torsion;

2. $t$ is the index in $E(K)$ of the group generated by $P^{+}$and $P^{-}$;

3. $W$ is the conjecturally finite Shafarevich-Tate group of $E$ over $K$;

4. $\Omega_{E}^{+}$is the real Néron period for $E / \mathbb{Q}$;

5. $c_{p}$ is the local Tamagawa factor attached to $E / \mathbb{Q}_{p}$.

Remark:

Note that the right-hand side in conjecture 3.1 can be re-written as

$$
\pm R_{\text {circle }}^{\frac{1}{2}} \cdot \sqrt{\# \amalg} \cdot \Omega_{E}^{+} \cdot \prod_{p \mid N} c_{p}
$$

and is analogous to the square root of the kind of expression in the leading term of $L(E / K, s)$ predicted by the classical Birch and Swinnerton-Dyer conjecture. This is clear for the Tamagawa factors (since all the primes dividing $N$ are split in $K / \mathbb{Q}$ ), for the factor $\Omega_{E}^{+}$(since $K$ is real quadratic) and for the term involving the order of the Shafarevich-Tate group. Finally it was explained in section 1 how $R_{\text {circle }}^{\frac{1}{2}}$ should be thought of as the "square root" of a hypothetical circle pairing regulator for $E(K)$.

In the case where $h^{+}>1$, let $H$ be the extension of $K$ which is cut out by $\chi$. Thus $H=K$ if $\chi$ is the trivial character, and $H$ is an unramified quadratic extension of $K$ otherwise.

Let $\sigma$ be a generator for $\operatorname{Gal}(H / K)$, and let

$$
\begin{aligned}
E(H)^{\chi} & :=\{P \in E(H) \text { such that } \sigma P=\chi(\sigma) P \text { for all } \sigma \in \operatorname{Gal}(H / K)\}, \\
W^{\chi} & :=\{\alpha \in W(E / H) \text { such that } \sigma \alpha=\chi(\sigma) \alpha \text { for all } \sigma \in \operatorname{Gal}(H / K)\}
\end{aligned}
$$

denote the $\chi$-parts of the Mordell-Weil group $E(H)$ and of the Shafarevich-Tate group of $E$ over $H$ respectively.

Choose a lift of $\tau$ to $\operatorname{Gal}(H / \mathbb{Q})$. Since $\tau$ commutes with $\operatorname{Gal}(H / K)$, it acts on $E(H)^{\chi}$, and one denotes by $E(H)^{\chi, \pm}$ the eigenspaces for this action, and by $r_{\chi, \pm}$ the corresponding ranks. Considerations involving the signs on the functional equation for $L(E / K, \chi, s)$ lead to the expectation that $E(H)^{\chi}$ has even rank, so that $r_{\chi}^{+}$and $r_{\chi}^{-}$should have the same parity.

The adèlic pairing

$$
\langle,\rangle: E(H) \times E(H) \longrightarrow C_{H}
$$


gives rise, by its functorial nature, to a pairing on $E(H)^{\chi}$ with values in $C_{K}$. Composing this pairing with $\eta: C_{K} \longrightarrow C_{K}^{-}$yields the corresponding circle pairing

$$
\langle,\rangle_{\text {circle }}: E(H)^{\chi} \times E(H)^{\chi} \longrightarrow C_{K}^{-},
$$

which satisfies properties similar to the case when $\chi$ is the trivial character. For example, both the submodules $E(H)^{\chi,+}$ and $E(H)^{\chi,-}$ are isotropic for this pairing.

For each rational prime $p \mid N$, let $\mathfrak{p}$ be a prime of $K$ above it and let $\sigma_{\mathfrak{p}}$ denote the frobenius element in $\operatorname{Gal}(H / K)$ attached to $\mathfrak{p}$. One may attach to $E$ and $\chi$ a Tamagawa factor $c_{p}^{\chi}$ by letting $c_{p^{2}}$ denote the Tamagawa factor attached to $E$ over the quadratic unramified extension of $\mathbb{Q}_{p}$, and setting

$$
c_{p}^{\chi}= \begin{cases}c_{p} & \text { if } \chi\left(\sigma_{\mathfrak{p}}\right)=1 \\ c_{p^{2}} / c_{p} & \text { if } \chi\left(\sigma_{\mathfrak{p}}\right)=-1\end{cases}
$$

Let $\Omega_{E}^{w}$ denote the real (resp. imaginary) Néron period for $E / \mathbb{Q}$ if $w=1$ (resp. $w=-1)$. The ratio $\left(\Omega_{E}^{w} / \Omega_{w}\right)$ is known to be a rational number. Let $d_{0}>0$ be its denominator, and let $d=d_{0} e$, where $e$ is as before the exponent of the torsion subgroup of $E(K)$. Let $C_{K}^{-}[d]$ denote the $d$-torsion subgroup of the group $C_{K}^{-}$.

Our conjecture can now be formulated as follows:

Conjecture 3.2 The derived period $J^{\prime}(f, K)$ is non-zero if and only if $E(H)^{\chi,+}$ and $E(H)^{\chi,-}$ both have rank one. In that case

$$
J^{\prime}(f, \chi) \cdot t \doteq \pm\left\langle P_{\chi}^{+}, P_{\chi}^{-}\right\rangle_{\text {circle }} \cdot \sqrt{\# W^{\chi}} \cdot\left(\Omega_{E}^{w} / \Omega^{w}\right) \cdot \prod_{p \mid N} c_{p}^{\chi} \quad \text { in } C_{K}^{-} / C_{K}^{-}[d],
$$

where

1. The symbol $\doteq$ denotes equality up to multiplication by a power of 2 ;

2. The points $P_{\chi}^{+}$and $P_{\chi}^{-}$are generators for $E(H)^{\chi,+}$ and $E(H)^{\chi,-}$ respectively, modulo torsion;

3. $t$ is the index of the group generated by $P_{\chi}^{+}$and $P_{\chi}^{-}$in $E(H)^{\chi}$.

Remark: Applying the natural projection

$$
C_{K}^{-} \longrightarrow C l^{+}(K)
$$

to conjecture 3.2 yields a variant of the conjectures of [MT2] for real quadratic fields which is spelled out precisely in conjecture 3.6 of [Da]. Thus conjecture 3.2 can be viewed as a (partial) lift of conjecture 3.6 of [Da] to the full idèle class group $C_{K}^{-}$, including the connected component of the identity of which class field theory does not provide a Galois-theoretic interpretation. 


\section{$4 \quad$ Numerical evidence}

We summarise some of the numerical evidence for conjecture 3.1 that has been gathered. Since the complexity of the period calculation increases with the size of the discriminant and fundamental unit of $K$, the experiments focused on the real quadratic fields of small discriminant $D=5$ and 13 .

Calculations with $\mathbb{Q}(\sqrt{5})$. Let $K=\mathbb{Q}(\sqrt{5})$ be the real quadratic field of discriminant 5 . It has narrow class number one, and its fundamental unit of norm one is given by

$$
\varepsilon_{K}=\frac{3+\sqrt{5}}{2} .
$$

There are exactly 3 elliptic curves of conductor $\leq 100$ with $\operatorname{sign}(E, \mathbb{Q})=-1$, all of whose prime factors are split in $\mathbb{Q}(\sqrt{5})$ : the curves denoted $61 \mathrm{~A}, 79 \mathrm{~A}$, and $89 A$ in the tables of Cremona. The equations for these curves, the coordinates for the points $P^{+}$and $P^{-}$, and the circle pairings for these points (with ten significant digits after the decimal point) are given below:

\begin{tabular}{|l|c|c|c|c|}
$E$ & Equation & $P^{+}$ & $P^{-}$ & $\left\langle P^{+}, P^{-}\right\rangle_{\text {circle }}$ \\
\hline $61 A$ & $y^{2}+x y=x^{3}-2 x+1$ & $(1,0)$ & $\left(\frac{4}{5}, \frac{-10+3 \sqrt{5}}{25}\right)$ & -0.9723644825 \\
$79 A$ & $y^{2}+x y+y=x^{3}+x^{2}-2 x$ & $(0,0)$ & $\left(\frac{1}{5}, \frac{-15+\sqrt{5}}{25}\right)$ & -0.4284370106 \\
$89 A$ & $y^{2}+x y+y=x^{3}+x^{2}-x$ & $(0,0)$ & $\left(\frac{-1}{5}, \frac{-10+7 \sqrt{5}}{25}\right)$ & -1.5571998775
\end{tabular}

(For the explicit formulae allowing the calculation of $\left\langle P^{+}, P^{-}\right\rangle_{\text {circle, }}$, see [MT1] or [Ca] for example.) The values of $t$ are readily calculated from the above table: one has $t=2,1$ and 2 for $E=61 A, 79 A$ and $89 A$ respectively.

To compute the derived period $I_{\Psi}^{\prime}$, we set

$$
F(z)=\int_{\infty}^{z} \omega_{f}=\sum_{n=1}^{\infty} \frac{a_{n}}{n} e^{2 \pi i n z}
$$

The assumption that $I(f, K)=0$ implies that $F(z)=F\left(\gamma_{\Psi} z\right)$ for all $z \in \mathcal{H}^{*}$, so that in particular $F\left(\gamma_{\Psi} \infty\right)=0$. Applying integration by parts:

$$
\int_{\infty}^{\gamma_{\Psi} \infty} \log \left(\frac{z-x_{\Psi}}{z-y_{\Psi}}\right) \omega_{f}=-\int_{\infty}^{\gamma_{\Psi} \infty} F(z) d \log \left(\frac{z-x_{\Psi}}{z-y_{\Psi}}\right) .
$$

Since the differential appearing on the right hand side of (23) is invariant under $\gamma_{\Psi}$, one can replace $\infty$ by any other $\tau \in \mathcal{H}^{*}$ in this expression without affecting its value. The optimal choice of $\tau$ is to take a value for which both $\operatorname{Im}(\tau)$ and $\operatorname{Im}\left(\gamma_{\Psi} \tau\right)$ are maximized:

$$
\tau=\frac{-d+i}{c}, \quad \text { so that } \gamma_{\Psi} \tau=\frac{a+i}{c}, \quad \text { where } \gamma_{\Psi}=\left(\begin{array}{cc}
a & b \\
c & d
\end{array}\right) .
$$


The table below lists the choices of $\gamma_{\Psi}$ that were made for the calculation of $I_{\Psi}^{\prime}=I^{\prime}(f, K)$, together with the value of the corresponding derived period.

\begin{tabular}{|c|c|c|c|}
$E$ & $61 A$ & $79 A$ & $89 A$ \\
\hline$\gamma_{\Psi}$ & $\left(\begin{array}{rr}19 & -5 \\
61 & -16\end{array}\right)$ & $\left(\begin{array}{rr}-28 & -11 \\
79 & 31\end{array}\right)$ & $\left(\begin{array}{rr}-8 & -1 \\
89 & 11\end{array}\right)$ \\
$I_{\Psi}^{\prime}$ & -0.0304845228 & -1.2747715571 & 1.6512851401
\end{tabular}

Finally, the table below summarises the calculations of the left and right hand sides occuring in conjecture 3.1. Here

$$
\begin{aligned}
& \text { LHS denotes } t \cdot I^{\prime}(f, K) \text {; } \\
& \# W^{\text {? }} \text { denotes the putative value of \# } W \text { that makes (22) hold; } \\
& \text { RHS denotes } \pm\left\langle P^{+}, P^{-}\right\rangle_{\text {circle }} \cdot \sqrt{\# W^{?}} \cdot \Omega^{+} \cdot \prod_{p \mid N} c_{p} \\
& \delta \quad \text { denotes }(L H S-R H S) /\left(\Omega^{+} \cdot \log \varepsilon_{K}\right) .
\end{aligned}
$$

\begin{tabular}{|l|r|c|r|r|}
$E$ & \multicolumn{1}{|c|}{ LHS } & $\# W^{?}$ & \multicolumn{1}{c|}{ RHS } & $\delta$ \\
\hline $61 A$ & -0.0609690456 & 1 & -5.9636991823 & 1 \\
$79 A$ & -1.2747715571 & 1 & -1.2747715571 & 0 \\
$89 A$ & 3.3025702803 & 1 & 8.6465494063 & -1
\end{tabular}

The last column in the table indicates that in these three calculations the quantity $\delta$ was always found to be an integer to within the calculated degree of accuracy, lending support for (but of course not proving) conjecture 3.1 in these examples. Although only 10 digits of numerical acuracy are indicated in the tables, the calculations were actually carried out to over 20 significant digits. The pari programs and script used to perform these calculations can be downloaded from the second author's web site.

Calculations with $\mathbb{Q}(\sqrt{13})$. Let $K=\mathbb{Q}(\sqrt{13})$ be the real quadratic field of discriminant 13. It has narrow class number one, and its fundamental unit of norm one is given by

$$
\varepsilon_{K}=\frac{11+3 \sqrt{13}}{2} .
$$

There are exactly 4 elliptic curves of conductor $N \leq 100$ with $\operatorname{sign}(E, \mathbb{Q})=-1$, all of whose prime factors are split in $\mathbb{Q}(\sqrt{13})$ : the curves denoted $43 A, 53 A$, $61 A$ and $79 A$ in the tables of Cremona. The equations for these curves, the coordinates for the points $P^{+}$and $P^{-}$, and the circle pairings for these points (with ten significant digits after the decimal point) are given below: 


\begin{tabular}{|l|c|c|c|r|}
$E$ & Equation & $P^{+}$ & $P^{-}$ & $\left\langle P^{+}, P^{-}\right\rangle_{\text {circle }}$ \\
\hline $43 A$ & $y^{2}+y=x^{3}+x^{2}$ & $(0,0)$ & $\left(\frac{61}{52}, \frac{-676+675 \sqrt{13}}{1352}\right)$ & 0.9717293862 \\
$53 A$ & $y^{2}+x y+y=x^{3}-x^{2}$ & $(0,0)$ & $\left(\frac{1}{13}, \frac{-91+25 \sqrt{13}}{13^{2}}\right)$ & -2.4475758590 \\
$61 A$ & $y^{2}+x y=x^{3}-2 x+1$ & $(1,0)$ & $\left(\frac{4}{13}, \frac{-26+31 \sqrt{13}}{13^{2}}\right)$ & 0.8669460262 \\
$79 A$ & $y^{2}+x y+y=$ & $(0,0)$ & $\left(\frac{-23}{13}, \frac{65+53 \sqrt{13}}{13^{2}}\right)$ & 0.7180776230 \\
& $x^{3}+x^{2}-2 x$ & & &
\end{tabular}

From this table it can be checked, by a direct calculation, that $t=2$ in all cases, i.e., the point $P^{+}+P^{-}$is always divisible by two in $E(K)$. The table below lists the derived period $I_{\Psi}^{\prime}$ in each case to ten digits of numerical accuracy, together with the value of $\gamma_{\Psi}$ used to compute it using (23).

$$
\begin{array}{|c|c|c|c|c|}
E & 43 A & 53 A & 61 A & 79 A \\
\hline \gamma_{\Psi} & \left(\begin{array}{rr}
40 & -9 \\
129 & -29
\end{array}\right) & \left(\begin{array}{rr}
-17 & -3 \\
159 & 28
\end{array}\right) & \left(\begin{array}{rr}
-65 & -27 \\
183 & 76
\end{array}\right) & \left(\begin{array}{rr}
-41 & -9 \\
237 & 52
\end{array}\right) \\
& & & \\
I_{\Psi}^{\prime} & 2.6570431602 & 5.4645636685 & -4.6691398640 & 2.1365682861
\end{array}
$$

The table below summarises the calculations of the left and right hand sides occuring in conjecture 3.1, with the same conventions as before:

\begin{tabular}{|l|r|c|r|r|}
$E$ & \multicolumn{1}{|c|}{ LHS } & $\# H^{?}$ & \multicolumn{1}{|c|}{ RHS } & $\delta$ \\
\hline $43 A$ & 5.3140863205 & 1 & 5.3140863205 & 0 \\
$53 A$ & 10.9291273370 & 1 & -11.4733570670 & 2 \\
$61 A$ & -9.3382797281 & 1 & 5.3171474282 & -1 \\
$79 A$ & 4.2731365722 & 4 & 4.2731365722 & 0
\end{tabular}

A calculation with the curve of conductor 5077. The elliptic curve $E$ of smallest conductor with rank 3 is given by the minimal Weierstrass equation

$$
y^{2}+y=x^{3}-7 x+6 .
$$

This curve has conductor 5077. The real quadratic field of narrow class number one with smallest discriminant in which 5077 splits is $K=\mathbb{Q}(\sqrt{53})$. It was checked for this field that the period $I^{\prime}(f, K)$ belongs to the lattice $\Lambda_{f} \log \varepsilon_{K}$. (In fact, in this computation the value of $I^{\prime}(f, K)$ turned out to be 0 to within the calculated accuracy of roughly 12 decimal digits.) This supports the prediction of conjecture 3.1 that the derived period $I^{\prime}(f, K)$ should be trivial whenever one of $r^{+}$or $r^{-}$is strictly greater than one. 


\section{A Gross-Kohnen-Zagier formula}

We begin by recalling a formula proved in [GKZ] which relates the height pairings between Heegner points coming from different imaginary quadratic fields to special values of certain $L$-series.

Given any (not necessarily fundamental) discriminant $D$, let $K_{D}=\mathbb{Q}(\sqrt{D})$ denote the corresponding quadratic field, let $H_{D}$ denote the ring class field attached to the order of discriminant $D$, and let $h_{D}$ denote the degree of $H_{D}$ over $K_{D}$.

In order to state theorem B of [GKZ] precisely it is useful to recall some of the notations used in this work. If $D<-4$ is a negative discriminant satisfying

$$
D \equiv r^{2} \quad(\bmod 2 N),
$$

then there are exactly $h_{D}$ distinct $\Gamma_{0}(N)$-orbits of integral binary quadratic forms $a x^{2}+b x y+c z^{2}$ of discriminant $D$ satisfying

$$
a>0, \quad a \equiv 0 \quad(\bmod N), \quad b \equiv r \quad(\bmod 2 N),
$$

the roots of which give representatives in $\mathcal{H} / \Gamma_{0}(N)$ for the distinct Heegner points in $X_{0}(N)\left(H_{D}\right)$ attached to the order of discriminant $D$, with orientation corresponding to the choice $r$ of square root of $D$ (modulo $N$ ). Let $\alpha_{D, r}$ be one of these points and let

$$
P_{D, r}=\sum_{\sigma \in \operatorname{Gal}\left(H_{D} / K_{D}\right)} \sigma \alpha_{D, r} \in \operatorname{Div}\left(X_{0}(N)\right)\left(K_{D}\right)
$$

denote the $K_{D}$-rational divisor of degree $h_{D}$ on $X_{0}(N)$ formed by taking the trace of $\alpha_{D, r}$. Finally let

$$
y_{D, r}=P_{D, r}-h_{D}(\infty)
$$

denote both the degree 0 divisor on $X_{0}(N)$, and, by a slight abuse of notation, its class in $J_{0}(N)\left(K_{D}\right)$. We write $P_{D, r}^{*}$ and $y_{D, r}^{*}$ for the images of $P_{D, r}$ and $y_{D, r}$ in $X_{0}^{*}(N)$ and $J_{0}^{*}(N)$ respectively, where $X_{0}^{*}(N)$ denotes the quotient of $X_{0}(N)$ by the Atkin-Lehner involution $W_{N}$ and $J_{0}^{*}(N)$ denotes its Jacobian.

Lemma 5.1 The element $y_{D, r}^{*}$ belongs to $J_{0}^{*}(N)(\mathbb{Q})$.

Proof: We already know that $y_{D, r}$ belongs to $J_{0}(N)\left(K_{D}\right)$. To analyse the action of $\operatorname{Gal}\left(K_{D} / \mathbb{Q}\right)=\langle\tau\rangle$ on $y_{D, r}$, note the equality of divisor classes on $X_{0}(N)$

$$
\tau y_{D, r}=W_{N} y_{D, r}+h_{D}((0)-(\infty)) .
$$

It follows immediately from this that the class of $y_{D, r}^{*}$ is fixed by $\tau$ and hence belongs to $J_{0}^{*}(N)(\mathbb{Q})$.

Let $\mathbb{T}$ be the subring of $\operatorname{End}\left(J_{0}^{*}(N)\right)$ generated by the Hecke operators $T_{p}$ $(p \nmid N)$ and $U_{q}(q \mid N)$. Fix a rational multiple $\mathbf{t}_{f} \in \mathbb{T} \otimes \mathbb{Q}$ of the idempotent attached to $f$. Such a $\mathbf{t}_{f}$ is only well-defined up to multiplication by a non-zero 
rational scalar, but one may choose to normalise $\mathbf{t}_{f}$ so that it belongs to $\mathbb{T}$ and is not divisible by any integer in $\mathbb{T}$. Let $\lambda_{f} \in \mathbb{Z}$ be the integer scalar defined by the rule

$$
\mathbf{t}_{f} f=\lambda_{f} f,
$$

and set $\left(y_{D, r}^{*}\right)_{f}:=\mathbf{t}_{f} y_{D, r}^{*}$

Finally define a number $d_{E}$ by the identity

$$
4 \pi^{2}\|f\|^{2}=d_{E} \Omega_{E}^{+} \Omega_{E}^{-} .
$$

It is known that $d_{E}$ is a rational number. In fact, if $E$ is the strong Weil curve of conductor $N$ it is equal (up to a possible factor of 2) to the degree of the minimal modular parametrisation attached to $E$. (Cf. the last equation in $\S 1$ of [Za].) Furthermore,

Lemma 5.2 The denominator of the rational number $\frac{\lambda_{f}}{2 d_{E}}$ divides $2 N^{i}$ for some $i$, and divides 2 if $N$ is prime.

Proof: The integer denoted $r$ in the proof of theorem 3 of [Za] clearly divides $\lambda_{f}$. The proof of theorem 3 (note, in particular, the last sentence) shows that $d_{E}$ divides $\lambda_{f} N^{i}$ for some exponent $i$ which can be taken to be 0 if $N$ is prime.

Let $D_{1}$ and $D_{2}$ be two negative coprime fundamental discriminants with $D_{i} \equiv r_{i}(\bmod 4 N)$. The product $D=D_{1} D_{2}$ is a positive fundamental discriminant, and the factorisation $D=D_{1} D_{2}$ corresponds, by genus theory, to an odd genus character

$$
\chi_{D_{1}, D_{2}}: \operatorname{Gal}\left(H_{D} / K_{D}\right) \longrightarrow\{ \pm 1\} .
$$

Recall the period $I\left(f, \chi_{D_{1}, D_{2}}\right)$ associated to $D$ and $\chi_{D_{1}, D_{2}}$ in equation (19).

Theorem B of [GKZ] states

$$
\left\langle\left(y_{D_{1}, r_{1}}^{*}\right)_{f}, y_{D_{2}, r_{2}}^{*}\right\rangle=\frac{\lambda_{f}}{2 d_{E}} \frac{I\left(f, \chi_{D_{1}, D_{2}}\right)}{i \Omega_{E}^{-}} \frac{L^{\prime}(E / \mathbb{Q}, 1)}{\Omega_{E}^{+}} .
$$

\section{Remark.}

1. The statement given here differs slightly from theorem B of [GKZ] in the way that we have grouped the terms, and in our definition of the $f$-isotypic elements $\left(y_{D_{i}, r_{i}}^{*}\right)_{f}$. In $[\mathrm{GKZ}]$, the element $\mathbf{t}_{f}$ is chosen to be an idempotent in $\mathbb{T} \otimes \mathbb{Q}$, so that $\lambda_{f}=1$. Requiring that $\mathbf{t}_{f}$ belong to the integral Hecke algebra $\mathbb{T}$ becomes essential for the analogue we wish to formulate next, in which the Néron-Tate height is replaced by the Mazur-Tate circle pairing on $J_{0}^{*}(N)(K)$ for a suitable real quadratic field $K$. This is because the circle pairing, whose value group is not uniquely divisible, does not extend naturally to $J_{0}^{*}(N)(K) \otimes \mathbb{Q}$.

2. Note that the first factor in the expression

$$
\frac{\lambda_{f}}{2 d_{E}} \cdot \frac{I\left(f, \chi_{D_{1}, D_{2}}\right)}{i \Omega_{E}^{-}}
$$


on the right in (26) is a rational number which is not far from being an integer, by lemma 5.2, and that the second factor is in fact an integer if the Manin constant attached to the modular parametrisation for $E$ is equal to 1 .

A circle pairing variant. We wish to express the circle pairing between two Heegner divisor classes in terms of a derived period integral. As before, we let $D_{1}$ and $D_{2}$ be negative fundamental discriminants, but in our setting are forced to relinquish the simplifying assumption that $D_{1}$ and $D_{2}$ are relatively prime. Rather, we must assume that $D_{1}$ divides $D_{2}$, so that

$$
D_{2}=d \cdot D_{1},
$$

where $d$ is a positive fundamental discriminant. Assume to simplify the discussion that the real quadratic field $K_{d}$ has narrow class number one.

As in the Gross-Kohnen-Zagier formula, let

$$
D=D_{1} D_{2}=d D_{1}^{2}
$$

This discriminant is not fundamental, but corresponds to the order of conductor $D_{1}$ in $\mathbb{Q}(\sqrt{d})$. Let $H_{D}$ be the ring class field attached to this order, and let $\chi_{D_{1}, D_{2}}$ denote the (generalised) genus character attached to the factorisation $D=D_{1} D_{2}$.

We define the Heegner element $y_{D_{1}, r_{1}}^{*}$ as in (25) and the sentence following it. Turning to the discriminant $D_{2}$, we let $\epsilon$ be the genus character of $K_{D_{2}}$ corresponding to the factorisation $D_{2}=d D_{1}$, which cuts out the quadratic extension $K_{D_{2}}(\sqrt{d})$ of $K_{D_{2}}$, and set

$$
y_{D_{2}, r_{2}, \epsilon}=\sum_{\sigma \in \operatorname{Gal}\left(H_{D_{2}} / K_{D_{2}}\right)} \epsilon(\sigma) \sigma \alpha_{D_{2}, r_{2}} \in \operatorname{Div}^{0}\left(X_{0}(N)\right)\left(K_{D_{2}}(\sqrt{d})\right) .
$$

By abuse of notation, we let $y_{D_{2}, r_{2}, \epsilon}$ (resp. $y_{D_{2}, r_{2}, \epsilon}^{*}$ ) denote the corresponding divisor classes in $J_{0}(N)\left(K_{D_{2}}(\sqrt{d})\right)$ (resp. in $J_{0}^{*}(N)\left(K_{D_{2}}(\sqrt{d})\right)$ ).

Lemma 5.3 The element $y_{D_{2}, r_{2}, \epsilon}^{*}$ belongs to $J_{0}^{*}\left(K_{d}\right)^{-}$.

Proof: The proof is similar to that of lemma 5.1 and is left to the reader.

Having in hand two explicit elements $y_{D_{1}, r_{1}}^{*}$ and $y_{D_{2}, r_{2}, \epsilon}^{*}$ in $J_{0}^{*}(\mathbb{Q})$ and $J_{0}^{*}\left(K_{d}\right)^{-}$ respectively, it is natural to ask for a formula for their Mazur-Tate circle pairing, in the spirit of the Gross-Kohnen-Zagier formula (26). Guided by conjecture 3.1, the following suggests itself naturally:

Conjecture 5.4 Let $m$ be the denominator of the rational number (27). Then

$$
\left\langle\left(y_{D_{1}, r_{1}}^{*}\right)_{f}, y_{D_{2}, r_{2}, \epsilon}^{*}\right\rangle_{\text {circle }}=\frac{\lambda_{f}}{2 d_{E}} \frac{I\left(f, \chi_{D_{1}, D_{2}}\right)}{i \Omega_{E}^{-}} \frac{I^{\prime}\left(f, K_{d}\right)}{\Omega_{E}^{+}} \quad\left(\bmod \mathbb{Z} \frac{\log \varepsilon_{K}}{m}\right) .
$$

This conjecture appears to be more tractable than conjecture 3.1. Since it only involves terms that are explicitly defined, one may hope that it would lend itself to an assault analogous to what is carried out in [GKZ]. The authors plan to return to this matter in a future publication. 


\section{References}

[Bo] E. D. Bone. The circle pairing on elliptic curves. Bachelor's thesis, Amherst College, 1995.

[BCDT] C. Breuil; B. Conrad; F. Diamond; R. Taylor. On the modularity of elliptic curves over $\mathbf{Q}$ : wild 3-adic exercises. J. Amer. Math. Soc. 14 (2001), no. 4, 843-939.

[BDG] M. Bertolini; H. Darmon; P. Green. Periods and points attached to quadratic algebras. to appear, in Proceedings of the MSRI workshop on special values of Rankin $L$-series, H. Darmon and S. Zhang, eds.

[BD1] M. Bertolini; H. Darmon. Heegner points on Mumford-Tate curves. Invent. Math. 126 (1996), no. 3, 413-456.

[Ca] G. Call. Local heights on families of abelian varieties. PhD Thesis, Harvard University, 1986.

[Da] H. Darmon. Heegner points, Heegner cycles, and congruences. Elliptic curves and related topics, 45-59, CRM Proc. Lecture Notes, 4, Amer. Math. Soc., Providence, RI, 1994.

[GKZ] B. Gross; W. Kohnen; D. Zagier. Heegner points and derivatives of L-series. II. Math. Ann. 278 (1987), no. 1-4, 497-562.

[Ha] S. R. Hamblen. Calculating the circle pairing over families of elliptic curves. Bachelor's thesis, Amherst College, 1998.

[Man] Ju. I. Manin. Parabolic points and zeta functions of modular curves. (Russian) Izv. Akad. Nauk SSSR Ser. Mat. 36 (1972), 19-66.

[MT1] B. Mazur; J. Tate. Canonical height pairings via biextensions. Arithmetic and geometry, Vol. I, 195-237, Progr. Math., 35, Birkhäuser Boston, Boston, MA, 1983.

[MT2] B. Mazur; J. Tate. Refined conjectures of the "Birch and SwinnertonDyer type". Duke Math. J. 54 (1987), no. 2, 711-750.

[Ne] A. Néron. Quasi-fonctions et hauteurs sur les variétés abéliennes. Ann. of Math. (2) 82 (1965) 249-331.

[Po] A. Popa. PhD Thesis, Harvard University. In progress.

[TW] R. Taylor; A. Wiles. Ring-theoretic properties of certain Hecke algebras. Ann. of Math. (2) 141 (1995), no. 3, 553-572.

[W] A. Wiles. Modular elliptic curves and Fermat's last theorem. Ann. of Math. (2) 141 (1995), no. 3, 443-551.

[Za] D. Zagier; Modular parametrizations of elliptic curves. Canad. Math. Bull. 28 (1985), no. 3, 372-384. 